\title{
College and University Library Statistics
}

$\bar{F}_{\mathrm{o}}^{\mathrm{o}}$ OR SEVERALL YEARS the February issue of the A.L.A. Bulletin has carried the statistics bf collegê, üniversity, and teachêrs college libraries. These statistics have been collected by the American Library Association Statistical Assistant and have been of much interest and value to librarians. Confronted by the necessity of retrenchment, the A.L.A. Executive Board decided to relieve the Headquarters staff of the necessity of compiling and publishing the statistics and to encourage the A.C.R.L. and its official organ, College and Research Libraries, to assume this responsibility. That was in October. Officers of the A.C.R.L. and the editors set to work promptly to work out a plan whereby the continuity of the statistics could be maintained.

With the reduction in the activities of the A.L.A. Statistical Assistant, it was necessary to seek volunteer assistance in preparing the material for publication. Julia Wright Merrill, chief of the A.L.A. Department of Information and Advisory Services, has aided greatly in the preparation and routing of material. The statistics for college and university libraries (Group I) were prepared and tabulated by Lucille Mock, loan department, Iowa State College Library, under the direction of Charles H. Brown, librarian. The statistics for college and university libraries (Group II) were prepared by G. Flint Purdy, librarian, Wayne University, Detroit. The statistics for teacher-training institutions were prepared by Clạra Guth- fie, feference librarian, Milner Library, Illinois State Normal University, and the staff of the Milner Library, of which Eleanor Weir Welch is librarian. All of these persons have very graciously accepted their share of the responsibility for getting the jol done efficiently and, it must be added, on rather short notice.

Because of the need for acotomy several items formerly included have ofent ofmitted from the statistics of the larger collese and university libraries (Group I) : numbier' of staff members on central library budget; salaries of administrative office assistants; salary scales for specific departments, cata$\log$, circulation, etc.; and student service rates. School and college libraries on central library budget and departmerital libraries on central library budget have been combined into one array of figures.

The items included for the small college and university libraries (Group II) and the teacher-training institutions are the same as those for the statistics of 1940-4I.

As is the case each year the list of institutions included varies from the list of previous years. Despite the various efforts that have been made in the past to continue the same libraries, some institutions failed to report and others reported too late. The variation this year is perhaps slightly greater, due no doubt to the effect of the war. To those libraries which have cooperated in spite of difficulties, College and Research Libraries extends thanks on behalf of all those who will use the statistics. 
Teachers College and Normal School Library General and Salary Statistics

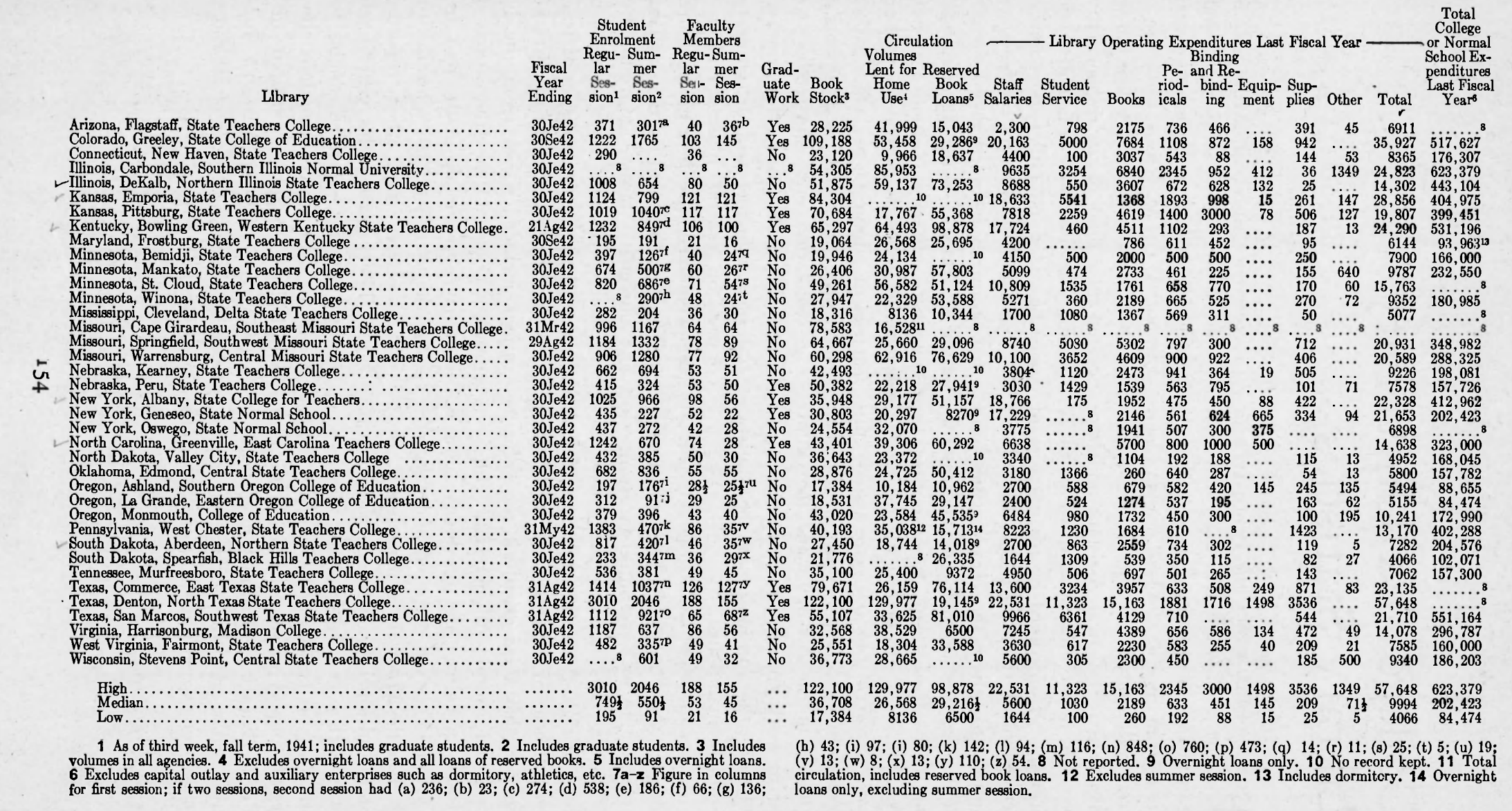


Teachers College and Normal School Library General and Salary Statistics

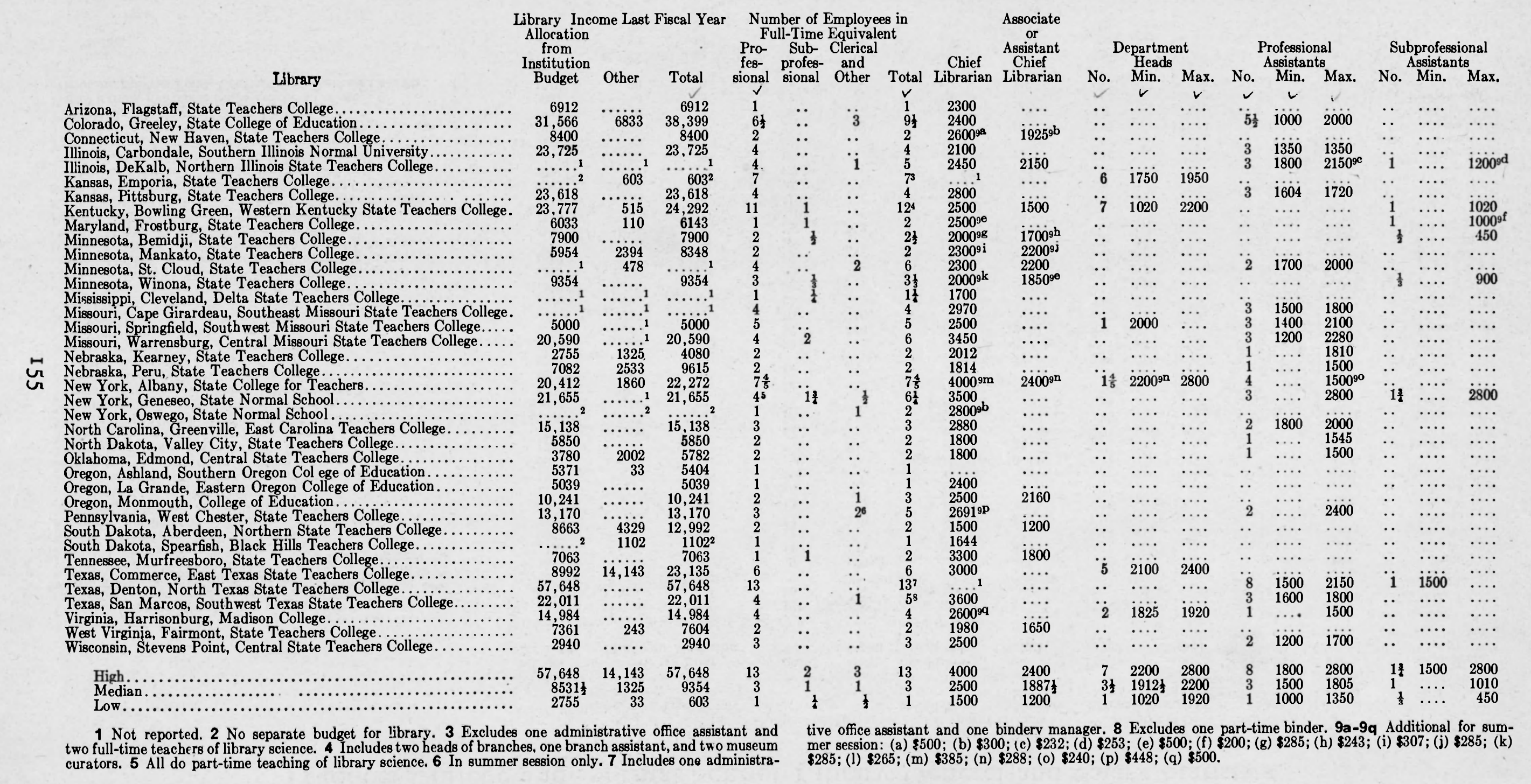


Teachers College and Normal School Library General and Salary Statistics

Library

Arlzona, Flagstaff, State Teachers College
Colorado, Greeley, State College of Education

Colorado, Greeley, State College of Education............

Illinois, Carbondale, Southern Illinois Normal University.

Illinois, DeKalb, Northern Illinois State Teachers College.

Kansas, Emporia, State Teachers College. ..

Kansas, Pittsburg, State Teachers College.......................

Kentucky, Bowling Green, Western Kentucky State Teachers College.

Minnesot, Bemidij, state Teachers College...

State Teachers Colleg.

Minnesota, St. Cloud, State Teachers College.

Misslssippi, Cleveland, Delta State Teachers College............

ज̆

Missour, Cape Girardeau, Southeast Missouri State Teachers Co o.lege.

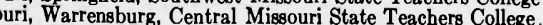

ebrack, Kearney, state Teachers College...

New York, Albany, State College for Teachers.

New York, Geneseo, State Normal School. .

orth Carolina, Greenville, East Carolina Teachers College

North Dakota, Valley City, State Teachers College......

Oregon, Ashland, Southern Oregon College of Education.............

Oregon, La Grande, Eastern Oregon College of Education.

Pennsylvania, West Chester, State Teachers College

south Dakota, Aberdeen, Northern State Teachers College.

South Dakota, Spearfish, Black Hills Teachers College.

Tennessee, Murreesboro, State Teachers College.

Texa, Denton, North Texas Ste Teachers College.

Texas, San Marcos, Southwest Texas State Teachers College.

Virginia, Harrisonburg, Madison College. ..........

Wisconsin, Stevens Point, Central State Teachers College.................<smiles>[141In]</smiles>

Low...

1 Not reported.

Approximate.

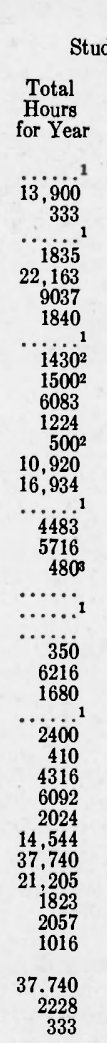

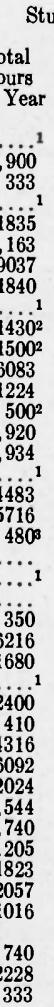

Number of Months for Hours per Week

Number of Months for Required of Each
Which Salary Is Paid Full-Time Staff Memb

Fiscal Year

Subpro-

$\begin{array}{ccc}\text { Rate per Hour } & \text { Profes- } \\ \text { Minimum Maximum } & \begin{array}{c}\text { and } \\ \text { and }\end{array}\end{array}$

Profes- fessional

Number of Days Allowed with Pay As:
Annual Vacation
Special Holidays

sional Clerical

$\begin{array}{ll}\dddot{30} & .40 \\ \cdots 1 & \cdots \\ \cdots 1 & \cdots\end{array}$

$\begin{array}{ll}.30 & .30 \\ .25 & .25\end{array}$

$\begin{array}{ll}.25 & .25 \\ .25 & .25 \\ . & .25\end{array}$

. $.11 \quad .35$

$.20 \quad .20$

$.25 \quad .25$

$.25 \quad .35$

.30

…

$\begin{array}{ll}\cdots 1 & \cdots 1 \\ .20 & .25 \\ .35 & .35 \\ .35 & .35 \\ .35 & .35 \\ .11 & .1{ }^{1} \\ .20 & .25 \\ .30 & \ldots 1 \\ .11 & .11 \\ .25 & .30 \\ .30 & .35 \\ .30 & .40 \\ .30 & .30 \\ .30 & .30 \\ .30 & .30 \\ .35 & .40 \\ .30 & .30 \\ .20 & .20\end{array}$

3 Summer session only.
Profes- $\begin{gathered}\text { fessional and } \\ \text { and }\end{gathered}$ Profes- $\begin{gathered}\text { sessional } \\ \text { and }\end{gathered}$

Cleric

Sabbatical

.........

….......

None
All
All

None

All

Profesiona

Professional
Professional

None

Professional

Librarian

.........

Libraria

Librarian

None

None

None

None
Professiona
None

$\begin{array}{llll} & 44 & 36 & 28 \\ 40 & 39 \frac{1}{2} & 22 \frac{1}{2} & 18 \\ 37 \frac{1}{2} & 15 & 12 & 10\end{array}$

4 Sixty hours during summer session.

........... 


\section{College and University Library General and Salary Statistics (Group I)*}

Library

Arizona........

Arkansas.....

Baylor.............................

Chicago........

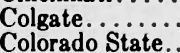

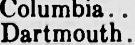

Duke........

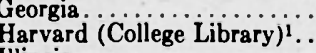

Iowa. .

Iowa State............................

Kansas........

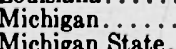

Minnesota.

Missouri ${ }_{\text {Mount Hooke. }}$

New York.

un North Dakota.

v

Oklahoma ${ }^{3}$

Princeton. . . .

Smith Dakota State

Syracuse..............

University Libraries. ...............
N.Y.State College of Forestry

Temple

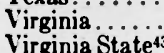

Washington (St. Louis).

Washington (St. Louis)

Wayne.........

Wyoming

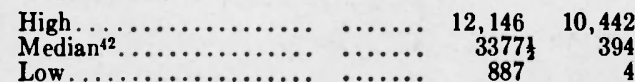

1 Includes main collection of Harvard College Library and fourteen noted. 2 Includes libraries in George Peabody College for Teachers, Searritt College for Christian Workers, and
Vanderbilt University. 3 Excludes Medical School and School of Nurges in Oklahoma City 4 Central library only except as otherwise noted. 5 As of third week, fall term, 1941 . 6 Includes graduate students. 7a-7u Figure
in column for first session; if two sessions, second session had (a) 444; (b) 229; (c) 600, includes duplicates; (d) 32

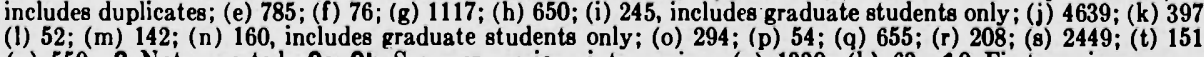
(u) 559. 8 Not reported. 9a-9b Surmer seesion; interseesion: (a) 1330; (b) 63. 10 First session; second seseion, 732; third session, 348. 11 Includes undergraduates in arts and music only. 12 First session; second
session, 4230; intersession, 437. 13 Approximate. 14 On four-quarter basis. 15 . On three-term basis. 16 In
Circulation.

Library Operating Expenditures Last Fisca

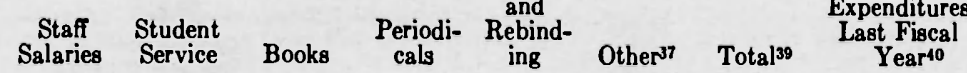

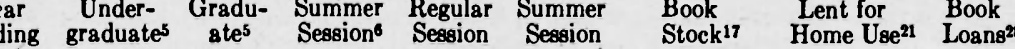

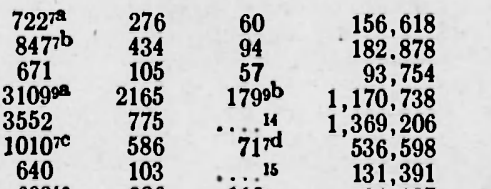

$\begin{array}{rr}34,434 & 92,494 \\ 40.042 & 134,354 \\ 9\end{array}$

$\begin{array}{rrrrrrr}17,465 & 3521 & 12,6843 & \ldots \ldots & 2922 & 1570 & 38.162 \\ 24,019 & 6078 & 14,544 & 3094 & 3080 & 1855 & 52,670\end{array}$ $\begin{array}{rrrrrrrrr}39,511 & 998127 & 10,925 & 9670 & 6731 & 1602 & 950 & 1621 & 31,499 \\ 205,114 & 260,88928 & 161,263 & 56,200 & 81,722 & 13,616 & 29,689 & 20,831 & 363,321 \\ & 157,293 & 35,214 & 47.617 & \mathbf{4 2 , 2 6 1} & 19,991 & 22,903 & 325,279 & \end{array}$

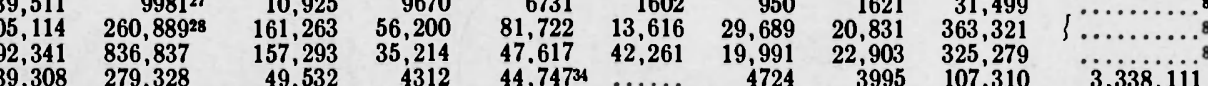
$\begin{array}{rrrrrrrrrr}7,916 & 28,222 & 14,200 & 1668 & 44859 & \cdots 2344 & 923 & 1515 & 28,509 & 3,338,1 i i\end{array}$

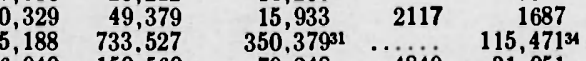

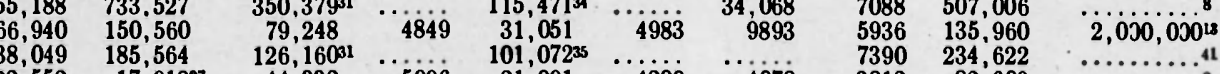

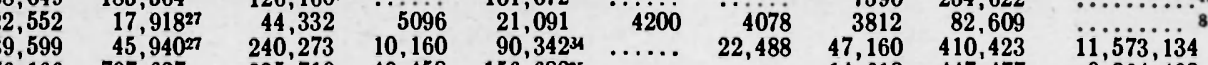

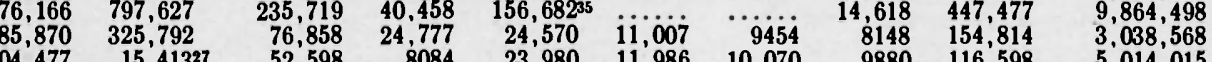
\begin{tabular}{llllllllll}
92.499 & 205,914 & 52,699 & 14,430 & 29,9184 & $\ldots .38$ & 7379 & 6303 & 110,729 & $\ldots \ldots \ldots \ldots .8$ \\
\hline & 121,208 & 30,748 & 10,476 & 18,388 & 6258 & 5625 & 3393 & 74,888 & $\ldots \ldots \ldots$
\end{tabular}

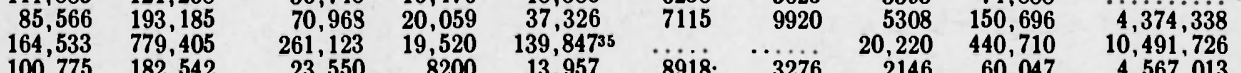

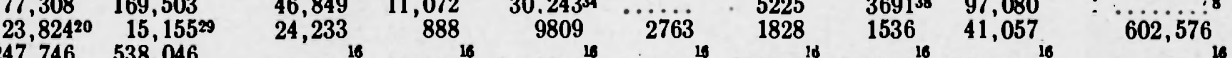

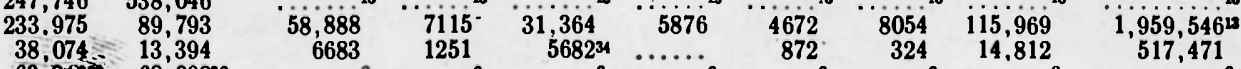

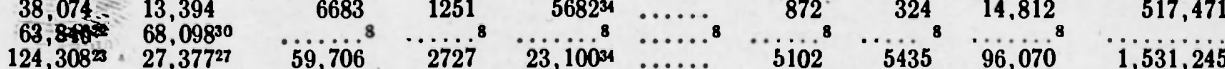
$\begin{array}{rr}84,373 & 271,886 \\ 68,025 & 124,451\end{array}$ $\begin{array}{cc}61,073 & 276,954 \\ 247,446^{24} & \end{array}$ $\begin{array}{lrlllll}59,706 & 2727 & 23,10034 & \ldots \ldots & 5102 & 5435 & 96,070 \\ 26,225 & 9723 & 22,5083 & \cdots \ldots & 1564 & 2596 & 62,616 \\ 42,226 & 12,132 & 17,353 & 4038 & 3038 & 4700 & 83,487\end{array}$ $\begin{array}{rrrrrrrr}48,567 & 13,369 & 13457 & 4038 & 3038 & 4700 & 83,487 & 1,050,711 \\ 75,3195 & 4178 & 83,524 & 2,620,837\end{array}$

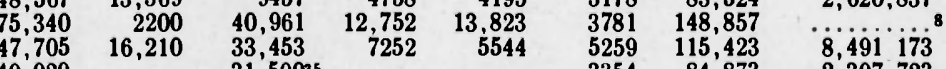

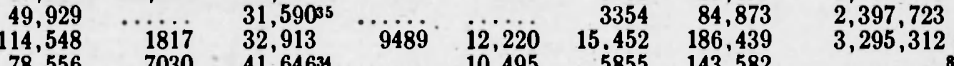
$\begin{array}{rrrrrrrr}78,556 & 7030 & 41,646^{34} & \ldots \ldots & 10,495 & 5855 & 143,582 & \cdots \\ 33,010 & 1381 & 15,31344 & \ldots \ldots & 1845 & 1391 & 52,940 & 11,726,191 \\ 6960 & 2105 & 3552 & \ldots 1689 & 893 & 911 & 16,110 & 1,199,869\end{array}$ $\begin{array}{rrrrrrrr}6960 & 2105 & 3552 & 1689 & 893 & 911 & 16,110 & 1,199,869 \\ 16,780 & 3405 & 11,657 & 2319 & 1375 & 1089 & 36,625 & 862,721 \\ 6 & & 19,957 & 6000 & 8232 & 2578 & \mathbf{9 8 , 2 9 5} & . \\ 6 & & & \end{array}$

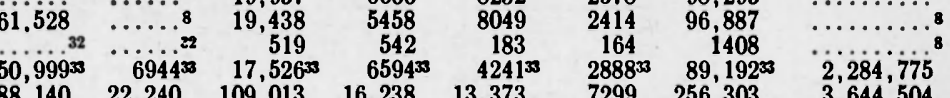
$\begin{array}{rrrrrrrr}88,140 & 22,240 & 109,013 & 16,238 & 13,373 & 7299 & 256,303 & 3,644,504 \\ 81,089 & 11,266 & 44,417 & 6254 & 7353 & 12,052 & 162,431 & 1,770,167 \\ 11,410 & 1990 & 2938 & 765 & 454 & 846 & 18,403 & 252,156\end{array}$ $\begin{array}{rrrrrrrr}11,410 & 1990 & 2938 & 765 & 454 & 846 & 18,403 & 252,156 \\ 24,180 & 2640 & 6494 & 6000 & 3890 & 2631 & 45,835 & \ldots \ldots \\ 75,860 & 22,847 & 17,076 & 9283 & 10,457 & 8061 & 143,584 & 3.068\end{array}$ $\begin{array}{rrrrcrrr}75,860 & 22,847 & 17,076 & 9283 & 10,457 & 8061 & 143,584 & 3,008,481 \\ 45,102 & 5120 & 27,670 & 5925 & 487936 & 1073 & 89,769 & 2,371,703 \\ 48,442 & 1573 & 16,972 & 4290 & 3291 & 3941 & 78,509 & 1,121,415\end{array}$ $\begin{array}{rlrlllrrrr}39,985 & 36,25527 & 48,442 & 1573 & 16,972 & 4290 & 3291 & 3941 & 78,509 & 1,121,415 \\ 55,661 & 73,619 & 14,544 & 2999 & 3514 & 2798 & 2895 & 3350 & 30,100 & 1,067,497 \\ 120,429 & 90,673 & 234,742 & 3349 & 93,1914 & \ldots . & 21,030 & 25,939 & 378,251 & 7,352,965\end{array}$ $\begin{array}{rlrrrrrrrr}655,188 & 836,837 & 261,123 & 56,200 & 109,013 & 42,261 & 34,068 & 47,160 & 507,006 & 11,573,134 \\ 84,373 & 129,402 & 49,049 \frac{1}{2} & 7030 & 17,957 & 59621 & 4801 & 3941 & 96,575 & 2,284,775 \\ 17,916 & 13,394 & 6683 & 888 & 1687 & 765 & 454 & 324 & 14,812 & 252,156\end{array}$ statistics. 20 Includes main library only. 21 Excludes overnight loans and all loans of reserved books. 22 Ining. 26 Includes overnight loans, 27 Includes overnight loans only. 28 Includes 23.725 sent 25 inside buildcount. 30 Excludes reserve books used from open shelves. 31 Includes student service. 32 Not paid from library budget. 33 Includes all libraries in institution. 34 Includes periodicals. 35 Includes periodicals and inding and rebinding. 36 Estimate. 37 Includes supplies. 38 Includes equipment. 39 Does not include enterprises such as dormitory, athletics, etc. 41 Not for publication. 42 In computing the medians only comparable figures were used, with the exception of the book stock figure for Harvard. 43 Retained in Group
I although undergraduate enrolment for 1941 is below 1000 . - Includes colleges and universities with enrol-
ments of over 1000 . 


\section{College and University Library General and Salary ${ }^{15}$ Statistics (Group I)*}

Library Income Last Fiscal Year

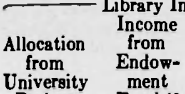

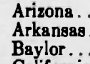

Californis (Berkeley)

Cincego.

Cagate...............

Dartmqut

Duke.

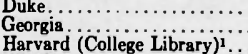

Illinois.

lowa State..............................

Kansas...

Louisiana.

Michigan ...........

Minnesota.......

Mount Holyoke.

North Carolina....

Northwestern.

$\stackrel{n}{\infty}$

Oberlin...

Oregon...................

Oregon State.............
Pennsylvania.

Pennsylvania Stat

Pittsburgh

ochester.

South Dakota Sta

outhern Methodist.

University Libraries ...............
N.Y.State College of Forestry

N.Y.S.
Temple.
Texas......
Virginis.

Virginis..............

Washington (St. Louig)

Weyne.:.
Wyoming
Wy

Yalet........

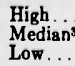

University
Budget
Funds ${ }^{10}$

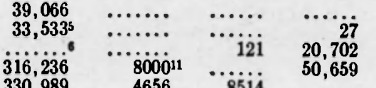

$\begin{array}{rr}330,989 & 469 \\ 27,600 & 28,58\end{array}$

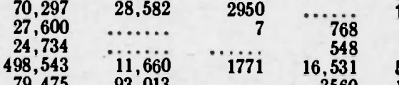

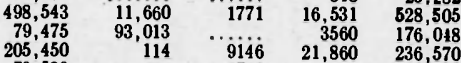

$\begin{array}{rrrrr}79,539 & 1674 & 9146 & 21,860 & 236,570 \\ 247,376 & 167,914 & 21,098 & 10,198 & 86,258 \\ & & 476,586\end{array}$

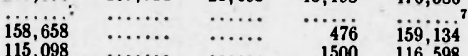

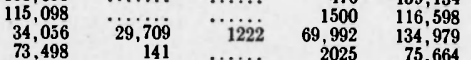

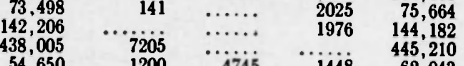

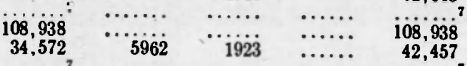

$\begin{array}{rrrrr}98,343 & 70,670 & \ldots \ldots . & \mathbf{8 8 0 7} & 117,880 \\ 14,175 & \ldots \ldots \ldots & \ldots \ldots & 430 & 14,605\end{array}$

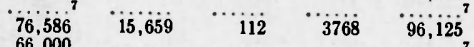

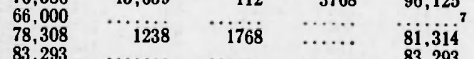

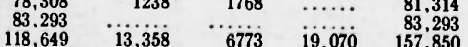

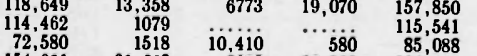

$\begin{array}{rrrrr}154,921 & 31,068 & 2085 & 18,380 & 85,088 \\ 129,126 & 18,337 & 1000 & 2803 & 151,266 \\ & 2461 & \ldots \ldots & 1017 & 3489\end{array}$

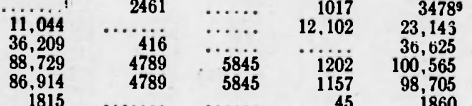

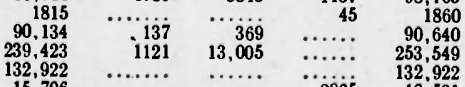

$\begin{array}{rrrrr}132,922 & \ldots \ldots \ldots & \ldots \ldots . & \ldots \ldots & 132.922 \\ 15,706 & \ldots \ldots \ldots & \ldots \ldots & 2825 & 18.531\end{array}$

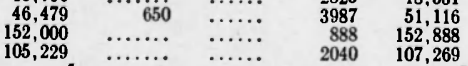

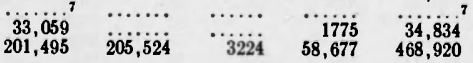

$\begin{array}{rrrrr}498,543 & 205,524 & 21,098 & 69,992 & 528,505 \\ 86,011 & 53754 & 2950 & 2803 & 104,559 \\ 11,044 & 114 & 7 & 27 & 14,605\end{array}$
Number of Employees in Full-Time Equivalent

Admin-
istrative
Office Office Clerical

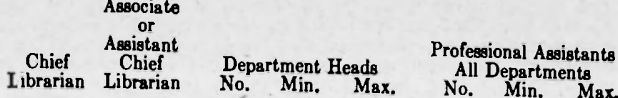

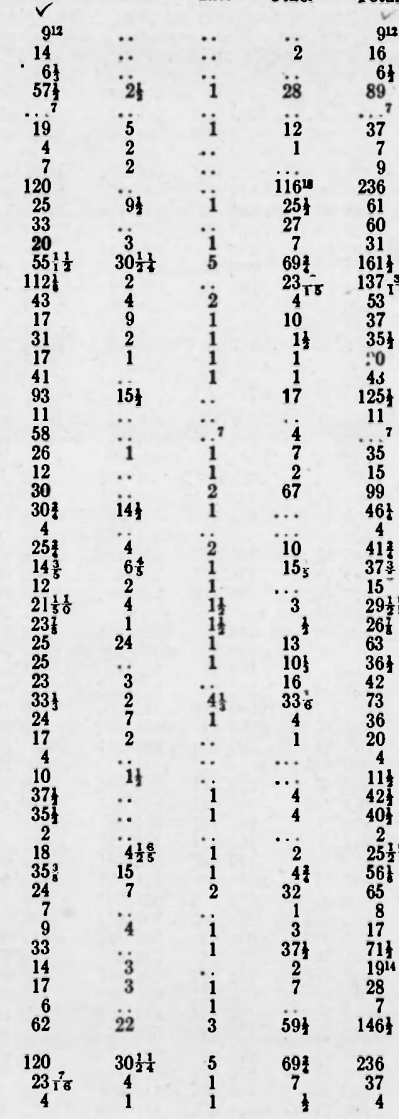

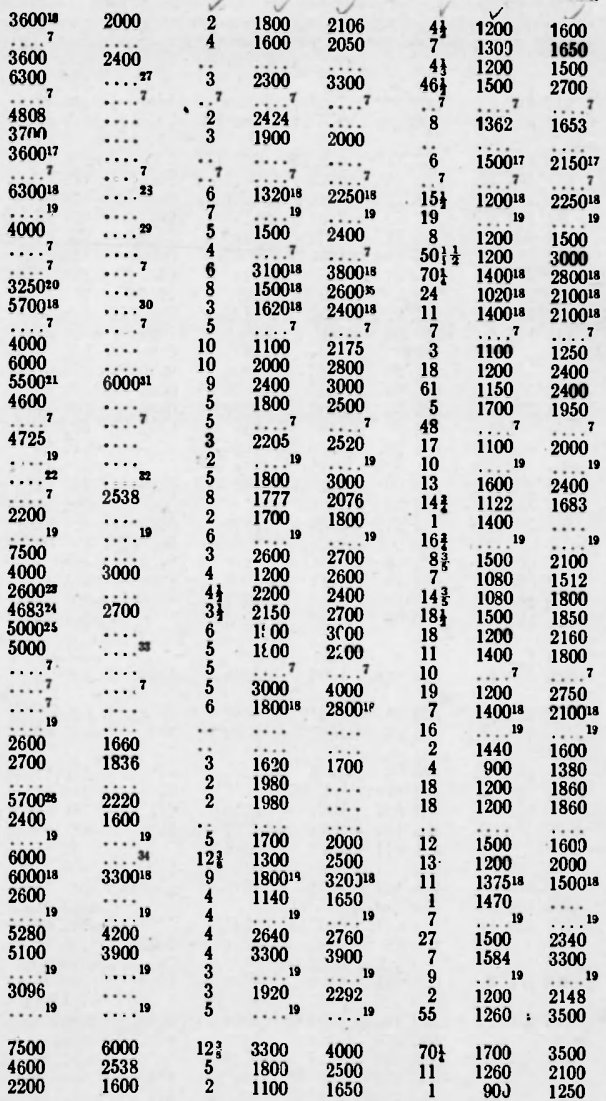

noted. 2 Includes librairestion of Harvaryard College Library and fourteen special libraries only unless otherwise and Vanderbilt University. 3 Excludes Medical School and School of Nurses in Oklahoma City. 4 Central
library only except as otherwise noted. 5 Estimate. 6 No separate budget for library. 7 Not reported. budget not reported. 10 Includes income from trust and all invested funds specifically deaignated university purposes. 11 Includes gifts. 12 Excludes one consulting librarian. 13 Includes subprofessional and adminisbeing paid as of Nov. 1, 1942. 16 Plus one consulting librarian at \$1700. 17 Salaries paid as of Nov. 1, 1941. 18 Salariea paid as of May 1 , 1942 . 19 Salary not for publication; or, confidential. 20 Serves as acting director,
assistant librarian, and also as a department head. 21 Balance of salary paid from budget of College of Litera

ture, Science, and the Arts. 22 Director of libraries at $\$ 10,000$. Also six librarians of individual libraries at
$\$ 1800$ to $\$ 6000$. 23 Plus director of libraries, Oregon State System of Higher Education, who is paid one eighth of salary by University of Oregon. 24 Receives one eighth of salary as director of libraries, Oregon State System of Higher Education. 25 Plus one director of libraries at one-half time. 26 Plus $\$ 500$ additional for summer 30 Two at $\$ 3200$ and $\$ 4200$. O and \$368 for summer session. 32 Three at $\$ 2700$ to $\$ 4700$. 33 Two at $\$ 250$ j and $\$ 2700$. 34 Two at $\$ 3400$ and 37 . 


\section{College and University Library General and Salary ${ }^{12}$ Statistics (Group I)*}

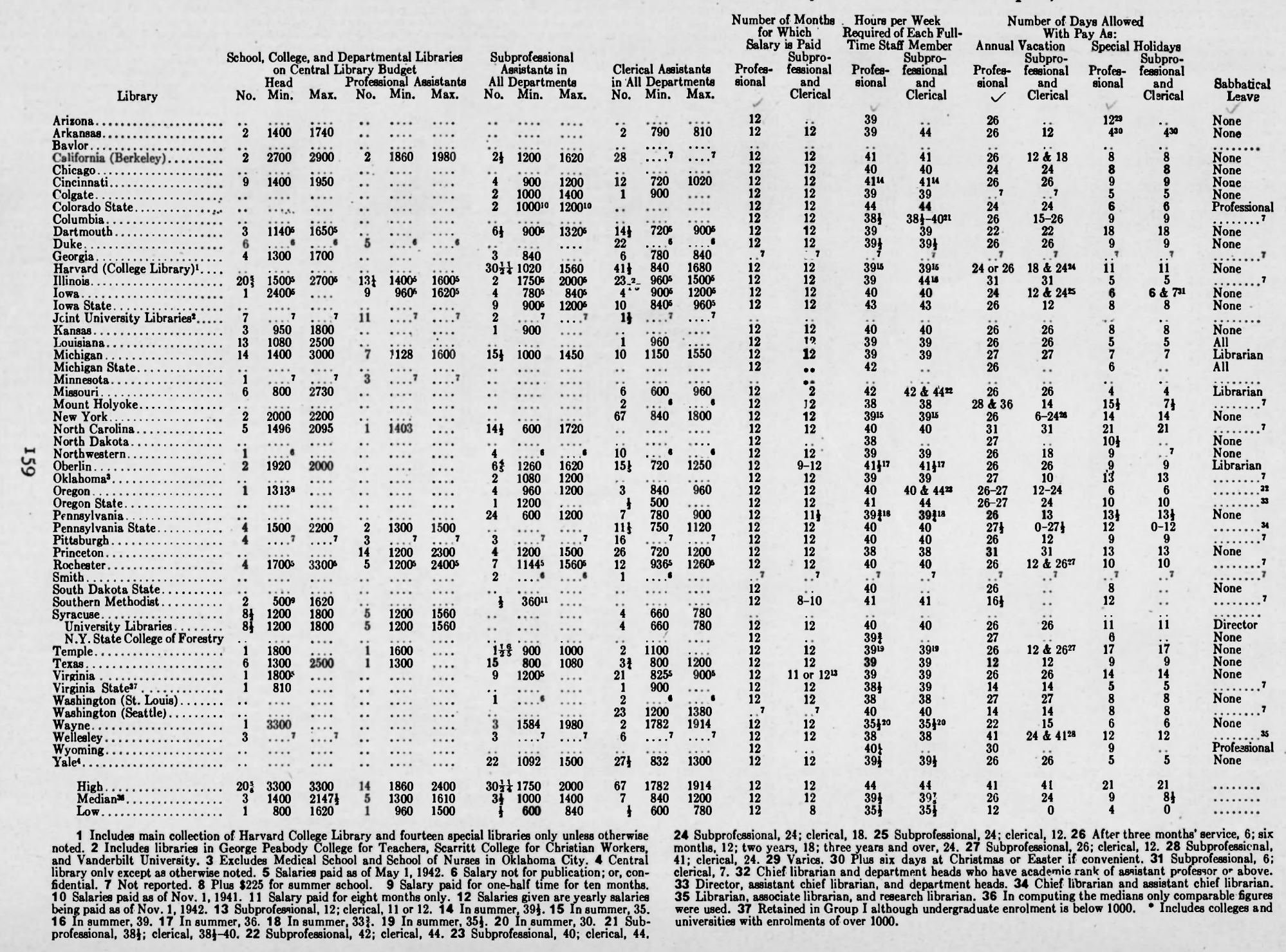




\section{College and University Library General and Salary Statistics (Group II)*}

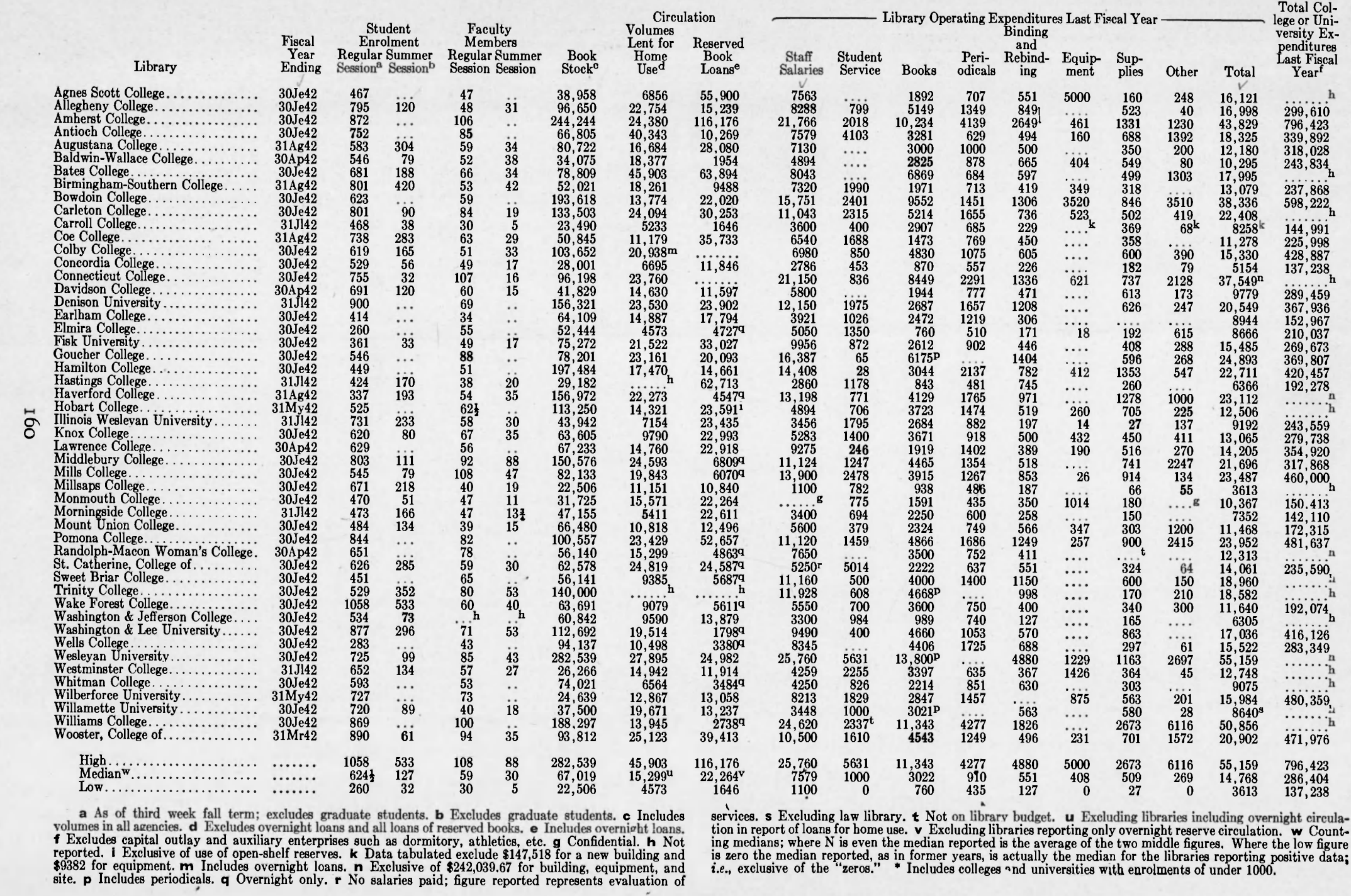




\section{Colleǵe and University Library General and Salary Statistics (Group II)*}

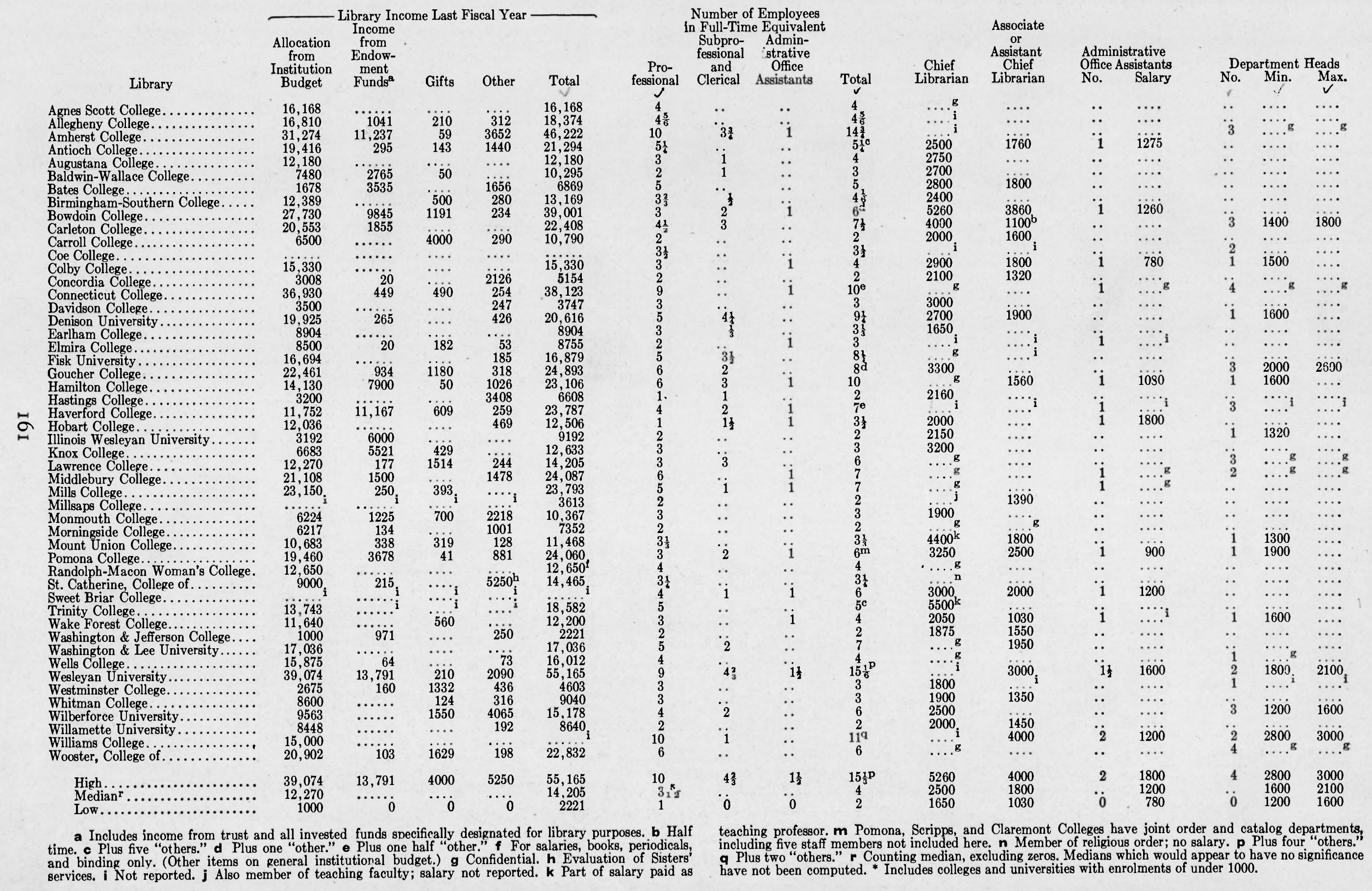




\section{College and University Library General and Salary Statistics (Group II)*}

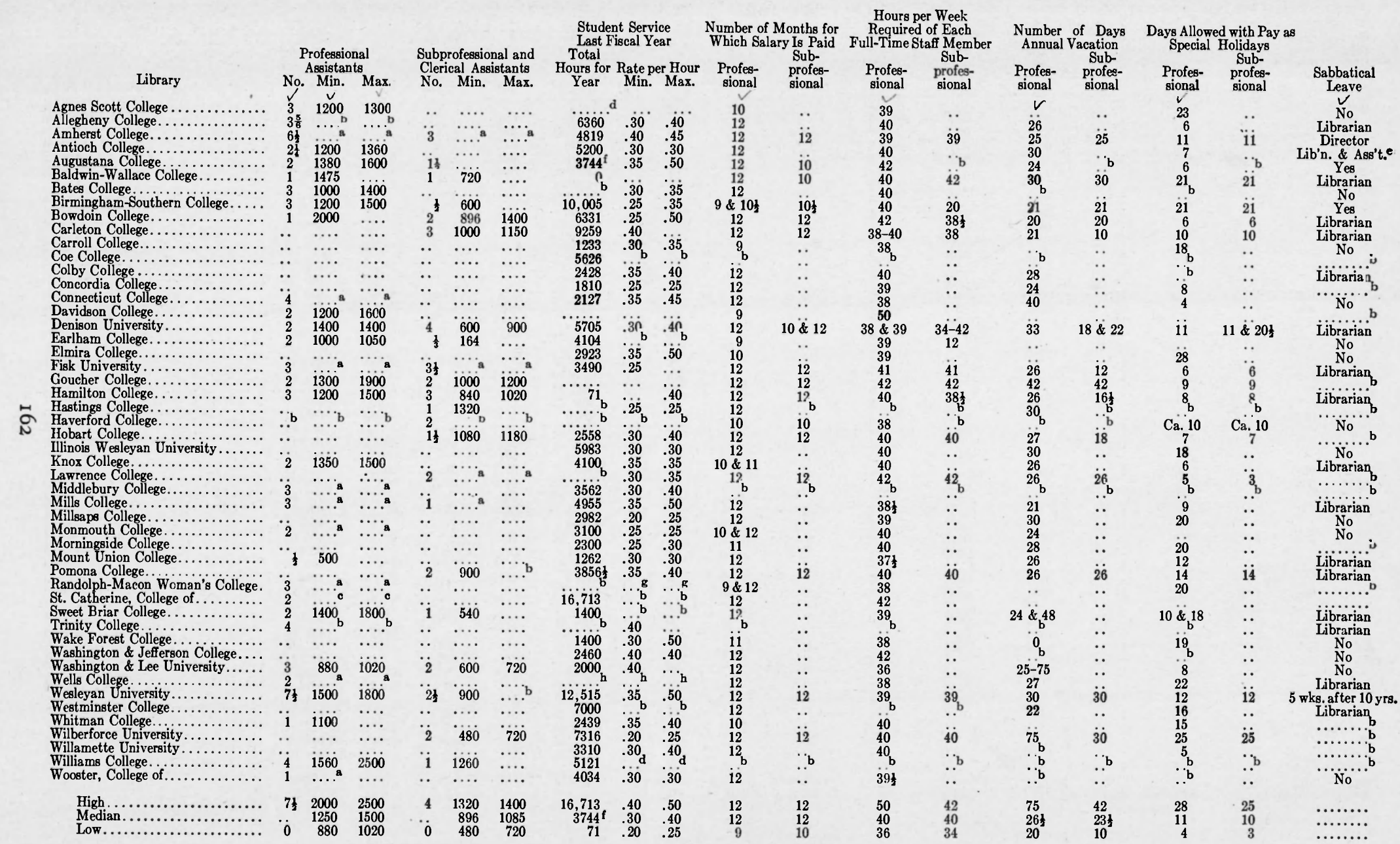

a Confidential. b Not reported.

a Confidential. b Not reported. $c$ Members of religious order; no salary. d Not paid from library budget.
e Librarian would receive 18 per cent of salary; assistant librarian 15 per cent. $f$ Including N.Y.A. $g$ Student 